\title{
Factores sociales relacionados con mala adherencia Antirretroviral en pacientes pediátricos. Hospital Mario Catarino Rivas
}

\author{
Social factors related to poor antiretroviral adherence at the Mario Catarino Rivas Hospital
}

Cristhian Mabel López Aguilar, * Luis Enrique Jovel López.

\section{RESUMEN}

Antecedentes: Adherencia es la capacidad del paciente de implicarse correctamente en la elección, inicio y control del tratamiento antirretroviral (TAR) permitiendo mantener el cumplimiento riguroso del mismo. Objetivo: determinar los factores socio-demográfico que intervinieron en mala adherencia al TAR en pacientes pediátricos infectados por $\mathrm{VIH}$ que asistieron al Centro de Atención Integral (CAI) del Hospital Nacional Mario Catarino Rivas (HNMCR), durante el período julio-septiembre 2015. Pacientes y Métodos: estudio cuantitativo, descriptivo, transversal. La población 724 pacientes menores de 20 años con VIH en TAR en el HMCR, muestra 31 pacientes con carga viral mayor de 1000 copias/ml y más de 6 meses de tratamiento, excluidos pacientes con resistencia primaria TAR documentada por genotipo, pacientes que no aceptaron participar en el estudio. Información recopilada mediante un instrumento tipo encuesta que incluía variables socio demográficas y tratamiento médico. Los datos fueron analizados con el programa EPIINFO y EXCEL. Resultados: se encontró que 13 (42\%) pacientes eran masculinos y 18 (58\%) femeninas. En cuanto a edad 20 (64\%) pacientes tenían entre 13-18 años. Se encontró que 27 (87\%) eran cuidados por familiares; madre 17 (63\%), padre $5(18 \%)$, abuelo $3(11 \%)$, primo 1 (4\%), tío 1 (4\%). Las razones de no adherencia fueron variadas en cada caso; 20 refirieron que por olvido, 7 porque el niño no aceptaba el medicamento. Conclusiones: los adolescentes

\footnotetext{
* Médico residente de tercer año del posgrado de Pediatría. Universidad Nacional Autónoma de Honduras, Valle de Sula, UNAH-VS. ** Pediatra profesor titular UNAH-VS.

Dirigir correspondencia a: cmlopez2012@yahoo.com Recibido: 30 de octubre 2015 Aprobado: 02 de marzo 2016
}

son los que presentaron mala adherencia al tratamiento, principalmente por olvido o no aceptar tomar el medicamento, hecho que puede estar influenciado por las relaciones interpersonales que se dan con otros jóvenes de su edad.

\section{PALABRAS CLAVE}

Pacientes, preparaciones farmacéuticas, VIH.

\section{ABSTRACT}

Background Adherence is the patient's ability to engage successfully in the election, initiation and control of antiretroviral therapy (ART) allowing to maintain strict compliance. Objetive: to determine the socio-demographic factors involved in poor adherence to ART in pediatric HIV-infected patients who attended the Comprehensive Care Center (CAI) of the Hospital Mario Catarino Rivas (HNMCR) during the period from July to September 2015. Patients and Methods: This is a quantitative, descriptive, cross-sectional study. There is a universe of 724 patients younger than 20 years with HIV on ART in HNMCR, there are 31 patients with viral load greater than 1000 copies / $\mathrm{ml}$ and more than 6 months of treatment. Patients with primary resistance TAR documented by genotype and patients who did not accept participate in the study were excluded. Information gathered through a survey that included socio-demographic and medical treatment variables. Data was analyzed with the EPIINFO and EXCEL software. Results: 13 (42\%) patients were male and 18 (58\%) female. $20(64 \%)$ patients had $13-18$ years of age. It was found that 27 (87\%) were cared for by relatives; mother 17 (63\%), father 5 (18\%), grandfather $3(11 \%)$, cousin 1 (4\%), Uncle 
1 (4\%). The reasons for no adherence were varied in each case; 20 reported that theyforgot to take the treatment and 7 reported that the child did not accept the drug. Conclusions: teenagers are the ones who often have poor adherence to treatment, mainly because they forgot to take the treatment or did not agree to take the drug. These reasons can be influenced by interpersonal relations that exist with others of their age.

\section{KEYWORDS}

Patient, Pharmaceutical Preparations, HIV.

\section{INTRODUCCIÓN}

El virus de inmunodeficiencia humana $(\mathrm{VIH})$ es el agente etiológico causante del síndrome de inmunodeficiencia humana adquirida que consiste en una afectación del sistema inmune a nivel de los linfocitos T CD4. ${ }^{(1)}$ A pesar de los grandes avances acontecidos en los últimos años, la infección avanzada por VIH constituye una de las principales causas de morbimortalidad a nivel mundial con afectación en la calidad de vida de los niños sobre todo en países en vías de desarollo. Para el 2008 a nivel mundial 2 millones de niños menores de 15 años vivían con la infección, en el 2013 esta cifra se había incrementado a 2,400,000 niños infectados. ${ }^{(2)}$

En Honduras, en el año 2010 existían 2280 casos, y para el 2014 el informe de ONUSIDA reportó 4978 casos. Las tres ciudades con mayor número de casos fue San Pedro Sula, Tegucigalpa, y La Ceiba. ${ }^{(3)}$

El tratamiento farmacológico es un aspecto determinante y fundamental para quienes viven con $\mathrm{VIH}$, por lo que en la última década del siglo XX comenzó a utilizarse el término adherencia. La adherencia se define como la capacidad del paciente de implicarse correctamente en la elección, inicio y control del TAR que permita mantener el cumplimiento riguroso del mismo con el objetivo de conseguir una adecuada supresión de la replicación viral, asegurando con esto la efectividad del tratamiento antirretroviral, aumentando las células CD4 y disminuyendo la carga viral. ${ }^{(4)}$ En la infección por VIH es indispensable una adherencia al tratamiento superior al 95\% para considerarla efectiva. Asegurando con esto el aumento de las células CD4 y disminuyendo la carga viral. En el caso de los niños, el cuidador primario cumple un papel fundamental dentro de su adherencia al tratamiento, participa en la toma de decisiones, supervisa y apoya la realización de las actividades de la vida diaria para compensar la disfunción existente en el niño con la enfermedad. ${ }^{(5)}$

Varios son los factores que pueden influir en una mala adherencia. Tenemos a los cuidadores, los pacientes y su edad, el sistema de salud y el esquema de tratamiento. Los factores asociados a los cuidadores son el olvido o falta de cumplimiento con el horario de la ingesta del medicamento, desconocimiento o creencias erróneas de la enfermedad y su tratamiento, horario de trabajo de los padres o cuidadores, falta de compromiso y responsabilidad por parte de los cuidadores, comentarios negativos que no favorecen la adherencia, el estrés de los padres o cuidadores, estado de salud física y mental de los cuidadores, consumo de alcohol o drogas de los padres o cuidadores, ausencia prolongada de padres o cuidadores. ${ }^{(6,7)}$ Los factores asociados a los pacientes son dificultad en la ingesta, (número y tamaño de las pastillas), imposibilidad de mantener la privacidad al momento de la ingesta, horario de la ingesta que interfiere con el sueño, estudios, juegos, comidas, dificultad para deglutir, mal sabor y olor fuerte de algunos medicamentos. ${ }^{(6,7)}$ La edad de los pacientes agrega situaciones especiales como casa en el horario de las tomas, temor a que los amigos puedan hacer preguntas, cansancio y agotamiento de las tomas diarias, vergüenza de tomar medicamentos frente a sus amigos, estrés y depresión, falta de educación acerca de la importancia de la adherencia al $\operatorname{TAR}^{(6,7)}$ 
En cuando al sistema de salud, la inadecuada relación entre el personal de salud, los familia res y los niños, niñas y adolescentes, el maltrato o discriminación por parte de los prestadores de salud, deficiencia en los servicios de salud, mala planificación en las consultas médicas, no disponibilidad y desabastecimiento de los medicamentos, proceso de consultas médicas engorroso y largo, el personal de salud no siempre es claro en las orientaciones relacionadas al TAR. ${ }^{(6,7)}$ Los factores asociados al esquema de tratamiento son horarios inadecuados o difíciles de llevar, dificultad para deglutir. (Sabor amargo), faltan medicamentos en versiones pediátricas, náuseas y vómitos, ardor en el estómago, diarreas, dolor. ${ }^{(6,7)}$

En la bibliografía consultada no se encontraron estudios publicados en nuestro país relacionados con la mala adherencia antirretroviral en pacientes con $\mathrm{VIH}$, es importante resaltar que las consecuencia que sufren estos pacientes repercuten en su calidad de vida. El objetivo fue determinar los factores socio-demográficos que intervinieron en la mala adherencia al tratamiento antirretroviral en pacientes pediátricos infectados por VIH que asistieron al centro de atención integral en el Hospital Mario Catarino Rivas durante julio a septiembre 2015.

\section{PACIENTES Y MÉTODOS}

Se realizó un estudio de enfoque cuantitativo, diseño descriptivo y de alcance transversal, en el CAI del HNMCR durante el período de julio a septiembre 2015. La población fue 724 pacientes menores de 20 años con VIH que asistieron por tratamiento antirretroviral en el período descrito. El tipo de muestreo fue por conveniencia obteniendo 31 pacientes. Se incluyeron todos los niños/as menores de 20 años en tratamiento antirretroviral que tenían carga viral mayor de 1000 copias/ml, con más de 6 meses de TAR y que firmaron el consentimiento informado. Se excluyeron pacientes fuera del rango de edad, con resistencia primaria a los
ARV documentada por genotipo y quienes no aceptaron participar en el estudio.

La técnica de recolección de datos fue la entrevista, y el instrumento fue un cuestionario que constaba de 36 preguntas abiertas y cerradas. Entre las características del paciente se incluyó la edad, procedencia, sexo, efectos adversos a los antirretrovirales, presencia de enfermedades oportunistas. En cuanto a las características del cuidador se interrogó sobre el vínculo, parentesco, edad, escolaridad, ingreso familiar, relación médico paciente. En cuanto a las características del tratamiento el régimen complicado, tratamientos adicionales, disponibilidad de los medicamentos. Se aplicó el cuestionario de adherencia modificado, donde se interrogó las variables de responsabilidad de administrar el medicamento, conoce su situación de VIH, cumplimiento con el horario del medicamento, razones de no adherencia.

Los datos obtenidos se procesaron en el programa estadístico EPIINFO versión 3.5.3. El análisis estadístico se hizo calculando medidas de frecuencia. Para organizar y presentar los datos se utilizó la hoja de cálculo Microsoft office Excel 2010.

Aspectos éticos: A cada participante se le explico el propósito del estudio y se solicitó su consentimiento informado para participar en el mismo, haciendo hincapié en que su participación era voluntaria y asegurando la confidencialidad y anonimato de la información.

\section{RESULTADOS}

Se encontró que de los 724 pacientes en TAR, 31 no tenían buena adherencia al tratamiento, haciendo una prevalencia de $4.28 \%$.

De los 31 casos, según la edad se encontró que 20 (64\%) de los pacientes estaban en el rango entre13-18 años. (Ver tabla No. 1) En cuanto a la distribución por sexo; 13 (42\%) eran masculinos y 18 (58\%) femeninos. 
Tabla No. 1: Rango de edad de pacientes con mala adherencia al tratamiento.

\begin{tabular}{lcc}
\hline Edad (años) & $\mathbf{n}$ & Porcentaje \\
\hline $0-6$ & 4 & $13 \%$ \\
$7-12$ & 7 & $23 \%$ \\
$13-18$ & 20 & $64 \%$ \\
Total & 31 & $100 \%$ \\
\hline
\end{tabular}

Fuente: Investigación mala adherencia en pacientes con VIH

En cuanto a la procedencia de los pacientes; 29 (94\%) eran del Departamento de Cortés, 1 (3\%) de Comayagua y 1 (3\%) del Departamento de Yoro.

Las personas que tenían la responsabilidad del cuidado de los pacientes con VIH eran; familiar 27 (87\%), 2 (6.5\%) niños eran cuidados en casa hogar y sin cuidador 2 (6.5\%). De los 27 niños que eran cuidados por un familiar, las madres eran las encargadas de 17 (63\%) de los niños. (Ver tabla No. 2)

Tabla No. 2: parentesco del cuidador del niño con VIH.

\begin{tabular}{lcc}
\hline Parentesco & $\mathbf{n}$ & Porcentaje \\
\hline Madre & 17 & $63 \%$ \\
Padre & 5 & $18 \%$ \\
Abuelo & 3 & $11 \%$ \\
Tío & 1 & $4 \%$ \\
Primo & 1 & $4 \%$ \\
Total & 27 & $100 \%$ \\
\hline
\end{tabular}

Fuente: Investigación mala adherencia en pacientes con VIH

Las razones para la mala adherencia al tratamiento en cada caso fueron múltiples; por olvido $35 \%$, el niño la rechazaba $12 \%$ y otros (madre deprimida, el niño no se encontraba en casa al momento de la toma, sueño) $18 \%$.

(Ver Tabla No. 3)
Tabla No. 3: Razones más frecuentes de la no adherencia al tratamiento en los niños con VIH.

\begin{tabular}{lcc} 
Razones de no adherencia & $\mathbf{n}$ & Porcentaje \\
\hline Se terminó la medicina & 3 & $5 \%$ \\
Olvido & 20 & $35 \%$ \\
El niño lo rechaza & 7 & $12 \%$ \\
$\begin{array}{l}\text { No desea que otros se } \\
\text { enteren }\end{array}$ & 3 & $5 \%$ \\
$\begin{array}{l}\text { El niño no estaba, cuando } \\
\text { debió tomar el medicamento }\end{array}$ & 2 & $4 \%$ \\
$\begin{array}{l}\text { Estaba ocupado } \\
\text { Cambio de rutina }\end{array}$ & 2 & $4 \%$ \\
Cuidador enfermó & 3 & $5 \%$ \\
$\begin{array}{l}\text { Medicamento con sabor } \\
\text { desagradable }\end{array}$ & 1 & $2 \%$ \\
$\begin{array}{l}\text { Preocupado por efectos } \\
\text { adversos }\end{array}$ & 4 & $7 \%$ \\
Otros & 2 & $4 \%$ \\
\hline Fute: & 10 & $18 \%$ \\
\hline
\end{tabular}

Fuente: Investigación mala adherencia en pacientes con VIH

\section{DISCUSION}

Los antirretrovirales se utilizan en el HNMCR desde 1996. Son medicamentos que disminuyen la multiplicación del $\mathrm{VIH} .{ }^{(8)}$ El tratamiento antirretroviral de gran actividad (TARGA) implica la combinación de tres o más drogas antirretrovirales de diferentes principios farmacológicos que permiten la disminución de la carga viral en sangre hasta niveles indetectables, conduciendo a la recuperación inmunológica del paciente en fase de inmunodeficiencia y ayudan a mejorar la calidad de vida proporcionando los siguientes beneficios: Reducen la cantidad del VIH en la sangre, lo que retarda el proceso de la enfermedad, restaura y preserva el sistema inmune, aumenta el número de linfocitos CD4, prolongan el tiempo de aparición de las enfermedades definitorias de sida, ayudan a que se desarrollen menos infecciones oportunistas, disminuye o evita las hospitaliza- 
ciones, mejora en la salud general y da capacidad para hacer más actividades diarias, reduce la morbilidad y mortalidad asociada a VIH, mantiene una supresión viral máxima y prolongada (carga viral indetectable idealmente), promueve o restaura el normal crecimiento $y$ desarrollo, y prolonga la sobrevida.(9)

En este estudio se encontró una prevalencia de $4.28 \%$ de no adherencia al tratamiento antirretroviral de gran actividad, lo cual indica que más del $95 \%$ de los pacientes con $\mathrm{VIH}$ atendidos en el HMCR se apegan al tratamiento $y$ presentan menos riesgo de morbi-mortalidad. Cifras alentadoras para el HNMCR pues otros estudios como el realizado en Tamaulipas México 2006- 2008, al evaluar la adherencia, se observó que el $50 \%$ no eran adherentes en las últimas cuatro semanas y un $48 \%$ en los últimos cuatro días de aplicada la encuesta. ${ }^{(10,11)}$

En este estudio el $64 \%$ de los pacientes que presentaban mala adherencia se encontraban en edades comprendidas entre 13 a 18 años, lo cual concuerda con estadísticas internacionales donde hay un aumento de posibilidades de no adherencia en un $10 \%$ por cada año que aumenta la edad. Esta es la razón por la cual sugieren prestar especial atención durante esta etapa; ya que son tratamientos adaptados de los complejos regímenes posológicos de los adultos, pero con los inconvenientes añadidos de que el adolescente dispone de menos autonomía, movilidad y privacidad. Además, existen problemas en el entorno social/familiar, baja autoestima, toxicomanías, cambios hormonales y metabólicos que hacen más prevalente la aparición de toxicidad. Adicional es el hecho de encontrarse en un estado avanzado de la infección por VIH, pueden conducir al abandono del tratamiento. ${ }^{(12-13)}$

Se encontró que la mayoría de los pacientes en el CAI-HNMCR eran cuidados por un familiar; el encargado era la madre $63 \%$ o el padre $18 \%$, haciendo un total de $81 \%$ que eran cuidados por sus progenitores. Según la literatura inter- nacional consultada, cuando el niño es cuidado por sus familiares hay mayor riesgo de mala adherencia sobre todo si está a cargo de familiares de primer grado su madre o padre, ya que son personas que no están psicológicamente preparados por su problema agregado. Considerando que en el caso de los niños, el cuidador primario cumple un papel fundamental dentro de su adherencia al tratamiento, el participa en la toma de decisiones, supervisa y apoya la realización de las actividades de la vida diaria para compensar la disfunción existente en la persona con la enfermedad. Para lograr una buena adherencia en estos pacientes es aconsejable que el cuidado sea llevado a cabo por una persona diferente a sus progenitores. $^{(14,15)}$

Además se encontró que $2(6.5 \%)$ niños eran cuidados en casa hogar y $2(6.5 \%)$ no tenían cuidador. En países donde la epidemia de sida es mayor el 15 al $20 \%$ de los niños son huérfanos de uno de sus padres, y algunos de ambos padres. En América Latina y el Caribe, las cifras de niñas y niños huérfanos a causa del SIDA han pasado en 1990 de 54,000 a más de 750,000 en $2005 .^{(5)}$

Las causas de no adherencia fueron múltiples en cada caso, pero la principal causa es el olvido $35 \%$, seguido de "el niño la rechazaba" $12 \%$. Estos resultados son similares a lo reportado en el estudio de Tamaulipas que reporta que las causas por las que los pacientes no se tomaron su medicamento en los últimos cuatro días fue el olvido $35 \%$ seguido de evitar los efectos desagradables $23 \% .{ }^{(10)}$ Según estadísticas internacionales los factores psicológicos es una de la principales razones de no adherencia al TAR, la cual aumenta una vez que el paciente se entera de su diagnóstico, y durante la etapa adolescente. ${ }^{(16)}$

En conclusión, el grupo de edad donde se presentó mala adherencia al tratamiento antirretroviral es la etapa de la adolescencia y los principales motivos son el olvido y no aceptar 
el medicamento. Además, en la mayoría de los casos la persona encargada del tratamiento del niño era un familiar de primer grado, principalmente la madre.

Se recomenda tratar en lo posible que el tratamiento antirretroviral se elija en función de disponibilidad de formas pediátricas, sabor agradable, simplicidad en las dosis, que interfiera lo menos posible con la actividad normal del niño. Y en cuanto a los padres fortalecer la educación de los tutores para estar mejor preparados y conscientes de la importancia que conlleva el cumplimiento del tratamiento.

\section{BIBLIOGRAFÍA}

1. Lamote Castillo JA. Infección por VIH/ sida en el mundo actual. Medisan. [Internet] 2014 [Citado 12 de noviembre 2015]; 18(7); 117- 138. Disponible en: http:// bvs. sld.cu/ revistas/san/vol18_7_14/san15714. pdf.

2. ONUSIDA. Informe sobre la epidemia mundial de sida. Catalogación por la Biblioteca de la OMS. "ONUSIDA/JC2502/1/S". 2013.

Disponible en: http://www.unaids.org/ sites/default/files/media_asset/UNAIDS _Global_Report_2013_es_1.pdf.

3. CONASIDA, Gobierno de la República de Honduras Secretaría de Salud, ONUSIDA. Resultados del Informe Nacional de Progreso de la Respuesta contra el VIH y el Sida. Honduras: 2015.

Disponible en: http://www.unaids.org/ sites/default/files/country/documents/HN D_narrative_report_2015.pdf.

4. OPS, OMS. Experiencias Exitosas en el Manejo de la Adherencia al Tratamiento Antirretroviral en Latinoamérica. Washinton D.C: NLM; 2011.

5. Ruiz Navia AM, Enriquez lara SL, Hoyos Hernández PA. Adherencia al tratamiento en niñas y niños con VIH. Pensamiento Psicológic. 2009;5(12):175-189.

6. Chirinos Cazorla P. Mejía Navarrete A, Cano Salinas J, Huamán Lozano S, Morán Zapata S, Liendo Cuenca L, et, al. Cuidados en el hogar y adherencia al tratamiento antirre- troviral de niños, niñas y adolescentes con VIH. Manual para capacitar a padres y cuidadores. Lima: Vía Libre; 2008.

Disponible en: http://www.portalsida.org/ repos/manual $\% 20$ cuidados $\% 20$ en $\%$ 20el\%20hogar\%20y\%20adherencia.pdf.

7. López López P. Actualización en infección por vih/sida en niños. Revista Gastrohnup. 2012; 14(1) Suplemento 1: S7-S18.

Disponible: http://revgastrohnup.univalle. edu.co/a12v14n1s1/a12v14n1s1art2.pdf.

8. Rodríguez de Schiavi M. Susana, Scrigni Adriana, García Arrigoni Patricia, Bologna Rosa, Barboni Graciela, Redondo Julia et al . Tratamiento antirretroviral de gran actividad en niños VIH positivos: Evolución de la enfermedad relacionada con parámetros clínicos, inmunológicos y virológicos al comienzo del tratamiento. Arch. argent. pediatr. [Internet]. 2009 Jun [citado 2016 Mar 01] ; 107( 3 ): 212-220. Disponible en: http://www.scielo.org.ar/scielo.php? script $=$ sci_arttext $\&$ pid $=$ S0325-0075200 9000300007\&lng=es.

9. Valdivia Vadella C, Soler Palacínb P, Martín-Nalda A, Cabañas Poy MJ, Clemente Bautista S, Espiau Guarnerb M, et al. Evaluación de un programa de valoración de adherencia al tratamiento antirretroviral. An Pediatr. [Internet] 2011 [Citado 10 noviembre 2015] ;75 (6):380-395.

Disponible en: https://dialnet.unirioja.es/ servlet/articulo?codigo $=3802378$. 
10. Peñarrieta MI, Kendall T, Martínez N, Rivera AM, Gonzales N, Flores F, et. al. Adherencia al tratamiento antirretroviral en personas con VIH en Tamaulipas, México. Rev. perú. med. exp. salud pública. [Internet] 2009 [Citado 20 de noviembre 2015]; 26(3). Disponible en: http://www.scielo.org.pe/ scielo.php?script=sci_arttext \&pid=S 1726-46342009000300011.

11. Xochihua-Díaz Luis. Apego al tratamiento antirretroviral en adolescentes. Bol. Med. Hosp. Infant. Mex. [revista en la Internet]. 2009 Ago [citado 2016 Mar 01] ; 66( 4 ): 306-313. Disponible en: http://www.scielo. org.mx/scielo.php?script=sci_arttext\&pid= S1665-11462009000400004\&Ing=es.

12. Panel de expertos del Colaborativo Español para la Infección VIH Pediatrica (CEVIHP), Sociedad Española de Infectología Pediátrica (SEIP) de la Asociación Española de Pediatría (AEP) y Secretaria del Plan nacional del Sida. Documento de consenso del CEVIHP/SEIP/AEP/SPNS respecto al tratamiento antirretroviral en niños y adolescentes infectados por el VIH. Actualización marzo 2012.

Disponible en: http://www.aeped.es/sites/ default/files/documentos/5_0.pdf.

13. Dilla Tatiana, Valladarez Amparo, Lizan Luis, Sacristan José Antonio. Adherencia y persistencia terapéutica: causas, consecuencias y estrategias de mejora. Atención
Primaria. [Internet] 2009 [Citado 09 de noviembre 2015];41(6):342-34813.

Disponible en: http://www.elsevier.es/es-re vista-atencion-primaria-27-articulo-adhe rencia-persistencia-terapeutica-causas-con secuencias-estrategias-mejora-13139004.

14. OPS, OMS. Tratamiento antirretroviral de la infección por el VIH en niños en Latinoamérica y el Caribe: en la ruta hacia el acceso universal, Recomendaciones para un enfoque de salud pública. Washington, D.C: NLM WC 503; 2008.

Disponible en: http://new.paho.org/hq/ dmdocuments/2008/GuiaARVninos.pdf.

15. Gobierno Bolivariano de Venezuela, Ministerio del Poder Popular para la Salud Venezuela. Reglamentación técnica para mejorar la adherencia al tratamiento antirretroviral en Venezuela. Caracas; 2008.

Disponible en: http://www.stopvih.org/ pdf/Reglamentacion_Tecnica_para_mejo rar_Adherencia_al_TARV_2008.pdf.

16. Trejos Ana María, Reyes Lizeth, Bahamon Marly Johana, Alarcón Yolima, Gaviria Gladys. Efectos en la adherencia al tratamiento y en el ajuste psicológico luego de la revelación del diagnóstico de VIH/SIDA con el modelo clínico "DIRE" en niños y jóvenes colombianos menores de 17 años. Rev. chil. infectol. 2015 Ago; 32(4): 408-415. DOI: http://dx.doi.org/10.4067/ S0716-10182015000500007. 